\title{
THE HILBERT METRIC ON TEICHMÜLLER SPACE AND EARTHQUAKE
}

\author{
Huiping Pan \\ Sun Yat-Sen University, School of Mathematics and Computational Science \\ 510275, Guangzhou, P. R. China; chnpanhp@foxmail.com
}

\begin{abstract}
Hamenstädt gave a parametrization of the Teichmüller space of punctured surfaces such that the image under this parametrization is the interior of a polytope. In this paper, we study the Hilbert metric on the Teichmüller space of punctured surfaces based on this parametrization. We prove that every earthquake ray is an almost geodesic under the Hilbert metric.
\end{abstract}

\section{Introduction}

Let $S_{g, n}$ be an orientable surface of genus $g$ with $n$ punctures. In this paper, we consider the surfaces of negative Euler characteristic with at least one puncture. A marked hyperbolic structure on $S_{g, n}$ is pair $(X, f)$ where $X$ is a complete hyperbolic metric on a surface $S$ and $f: S_{g, n} \rightarrow S$ is a homeomorphism. Two marked hyperbolic structures $\left(X_{1}, f_{1}\right)$ and $\left(X_{1}, f_{2}\right)$ are called equivalent if there is an isometry in the isotopy class of $f_{1}^{-1} \circ f_{2}$. For simplicity, we usually denote a marked hyperbolic metric by $X$ instead of the pair $(X, f)$. The Teichmüller space $T_{g, n}$ is defined as the space of equivalent classes of marked hyperbolic structures on $S_{g, n}$. It is well known that $T_{g, n}$, equipped with the natural topology is homeomorphic to a ball in $\mathbf{R}^{6 g-6+2 n}$.

Given an open convex domain $D \subset \mathbf{R}^{m}$, Hilbert defined a natural metric on $D$, now called the Hilbert metric, such that the straight line segments are geodesic segments under this metric. In [15], Papadopoulos raised a problem: "Realize Teichmüller space as a bounded convex set somewhere and study the Hilbert metric on it". In this paper, we will study this problem.

For the case of closed surfaces, Yamada [29] constructed a space which he called the Teichmüller-Coxeter complex within which the original Teichmüller space sits as an open convex but unbounded subset, based on the Weil-Petersson completion of the Teichmüller space. Then in [30], after introducing a new variational characterization of the Hilbert metric, he defined the Weil-Petersson Hilbert metric on the Teichmüller space, where the background geometry is the one induced by the Weil-Petersson geometry instead of the Euclidean geometry.

For the case of punctured surfaces, Hamenstädt [5] provided a parametrization of the Teichmüller space into $\mathbf{R} P^{6 g-6+2 n}$ by length functions such that the image of $T_{g, n}$ is the interior of a finite-sided polyhedron (see §2.3). Therefore the Hilbert metric is well defined on $T_{g, n}$. Hamenstädt's parametrization depends on the choice of a preferred triangulation $\Gamma$ of $S_{g, n}$. More precisely, fix a puncture of $S_{g, n}$ and denote it as $O$, let $\Gamma=\left\{\eta_{1}, \eta_{2}, \ldots, \eta_{6 g-5+2 n}\right\}$ be a set of bi-infinite simple curves on $S_{g, n}$ such that for any marked hyperbolic metric $X$ the two ends of $\eta_{i}, i=1,2, \ldots, 6 g-5+2 n$, go into the puncture $O$ and such that $S_{g, n} \backslash \Gamma$ consists of $4 g-3+n$ ideal triangles 
and $n-1$ once punctured discs. Such a set $\Gamma$ is called a preferred triangulation of $S_{g, n}$. There are countably many choices of preferred triangulations.

In this paper, we study the Hilbert metric $d_{h}^{\Gamma}$ on $T_{g, n}$ based on Hamentädt's parametrization. Before stating our main result, we briefly explain a deformation of hyperbolic metric introduced by Thurston in [23], namely, the earthquake.

Let $\alpha$ be a simple closed curve on $S_{g, n}$, and $X \in T_{g, n}$ be a marked hyperbolic metric. Denote by $\alpha^{*}$ the geodesic representative of $\alpha$ on $X$. Cutting $X$ along $\alpha^{*}$ and twisting to the left about distance $t$, we obtain a new marked hyperbolic metric, denoted by $\mathcal{E}_{\alpha}^{t} X$. Note that the notion of "left" twist depend only on the orientation of $X$ (no orientation of $\alpha^{*}$ is necessary). Thurston extended this construction to any measured geodesic lamination. He proved the following result, one of whose proof can be found in [9].

Proposition 1.1. There is a (unique) continuous map $\mathcal{M L} \times \mathbf{R} \times T_{g, n} \rightarrow T_{g, n}$, associating an element $\mathcal{E}_{\alpha}^{t} X \in T_{g, n}$ to $(\alpha, t, X)$, such that $\mathcal{E}_{\lambda \alpha}^{t} X=\mathcal{E}_{\alpha}^{\lambda t} X$ for all $\lambda>0$ and all $\alpha \in \mathcal{M L}$, and such that when $\alpha$ is a simple closed geodesic, $\mathcal{E}_{\alpha}^{t} X$ is obtained from $X$ by the earthquake defined above.

The metric $\mathcal{E}_{\alpha}^{t} X$ defined in Proposition 1.1 is said to be obtained from $X$ by a (left) earthquake of amplitude $t$ along the measured geodesic lamination $\alpha$, and the orbits $\left\{\mathcal{E}_{\alpha}^{t} X\right\}_{t=-\infty}^{\infty},\left\{\mathcal{E}_{\alpha}^{t} X\right\}_{t=-\infty}^{0}$ and $\left\{\mathcal{E}_{\alpha}^{t} X\right\}_{t=0}^{\infty}$ are called the earthquake line directed by $\alpha$ and starting at $X$, the anti-earthquake ray directed by $\alpha$ and starting at $X$, and the earthquake ray directed by $\alpha$ and starting at $X$, respectively.

Recall that for a metric space $(X, d)$, an unbounded path $\gamma:[0, \infty) \rightarrow X$ is called an almost-geodesic if for any $\epsilon>0$, there exists $T>0$, such that

$$
\mid d(\gamma(0), \gamma(s))+d(\gamma(s), \gamma(t))-t) \mid<\epsilon
$$

for any $t \geq s \geq T$.

Now we state our main result.

Main Theorem. After reparametrization, every (anti-)earthquake ray is an almost-geodesic in $\left(T_{g, n}, d_{h}^{\Gamma}\right)$.

In fact, the image of an earthquake ray under Hamenstädt's parametrization eventually looks like a projective line (see §3).

Outline. This paper is organized as the following. In Section 2, we recall some basic properties of the Hilbert metric and express the Hilbert metric $d_{h}^{\Gamma}$ on the Teichmüller space based on Hamenstädt's parametrization. In Section 3, we prove our main theorem. In Section 4, we study the dependence of the Hilbert metric $d_{h}^{\Gamma}$ on the choice of the preferred triangulation $\Gamma$. We will show that a sphere $B\left(X_{0}, R\right)$ centered at $X_{0} \in T_{g, n}$ of radius $R$ with respect to $d_{h}^{\Gamma}$ for a preferred triangulation $\Gamma$ is again a sphere up to an additive constant with respect to $d_{h}^{\Gamma^{\prime}}$ for another preferred triangulation $\Gamma^{\prime}$, provided that $\Gamma^{\prime}$ can be obtained from $\Gamma$ by a diagonal-flip (to be defined in $\S 4)$. But the additive constant depends on the center point $X_{0}$. In Section 5 , we study the actions of the mapping class group on $\left(T_{g, n}, d_{h}^{\Gamma}\right)$. It is well known that when the Teichmüller space is endowed with the Teichmüller metric, the Thurston metric or the Weil-Petersson metric, the mapping class group acts by isometries. But here, we will show that the action of a positive Dehn twist is not isometric (see Corollary 5.4). Instead, it is an almost isometry on an unbounded subset of $\left(T_{g, n}, d_{h}^{\Gamma}\right)$ (see Corollary 5.2). 
Acknowledgements. I would like to thank my advisor Lixin Liu for his careful reading of this manuscript and many useful suggestions. I would like to thank Athanase Papadopoulos for useful conversations during his visit at Sun Yat-Sen University (Zhongshan University). I also thank the referee for numerous comments and suggestions. The work is partially supported by NSFC, No:11271378.

\section{The Hilbert metric on the Teichmüller space}

2.1. The Hilbert metric. There are two versions of the Hilbert's metric. The first version is the original one due to Hilbert which is defined on a bounded convex domain $\Omega$ (see Fig. 1(a)) in $\mathbf{R}^{m}$. Let $x, y$ be two points in the interior of $\Omega$, the line passing through $x, y$ intersects the boundary $\partial \Omega$ at two points $a, b$, where $x$ lies between $a$ and $y$. Then the Hilbert metric is defined as:

$$
d_{H}(x, y)=\frac{1}{2} \log [a, b, y, x]=\frac{1}{2} \log \frac{|a-y||b-x|}{|a-x||b-y|},
$$

where $[a, b, y, x]$ represents the cross-ratio of $a, x, y, b$.

The second version is due to Birkhoff which is defined on the cone $\mathcal{C}$ over a bounded convex domain $\Omega$ (see [11], [10] and [12] for more details about this version). Recall that a cone $\mathcal{C}$ is called pointed if $\mathcal{C} \cap-\mathcal{C}=0$. Let $\mathcal{C}$ be a closed, pointed (convex) cone over a convex bounded domain $\Omega$ in $\mathbf{R}^{m}$. Given two nonzero vectors $x$ and $y$ in $\mathcal{C}$ (see Fig. 1(b)), the Birkhoff's version of the Hilbert metric, denoted as $d_{h}$ is defined as:

$$
d_{h}(x, y)=\frac{1}{2} \log M(x, y) / m(x, y)
$$

where

$$
\begin{aligned}
M(x, y) & =\inf \{\lambda \geq 0: \lambda y-x \in \mathcal{C}\} \\
m(x, y) & =\sup \{\lambda \geq 0: x-\lambda y \in \mathcal{C}\} .
\end{aligned}
$$

Denote by $o$ the cone point of $\mathcal{C}$, and suppose that the line $\overline{x y}$ passing through $x, y$ intersects the boundary $\partial \mathcal{C}$ at $a, b$. To calculate $M(x, y)$ explicitly, we distinguish two cases. The first case is that the points $o, y, x$ are collinear. In this case $M(x, y)=$ $m(x, y)=|x| /|y|$, hence $d_{h}(x, y)=0$. The second case is that the points $o, x, y$ are not collinear. We draw an auxiliary line $\overline{x p}$ from $x$ which is parallel to the line $\overline{o b}$ and intersects the line $\overline{o y}$ at $p$. Then

$$
M(x, y)=\frac{|p-o|}{|y-o|}=\frac{|x-b|}{|y-b|} .
$$

Similarly we get

Hence

$$
m(x, y)=\frac{|x-a|}{|y-a|}
$$

$$
\frac{M(x, y)}{m(x, y)}=\frac{|a-y||b-x|}{|a-x||b-y|}=[a, b, y, x] .
$$

By the property of cross-ratio, we have $d_{h}(\lambda x, \mu y)=d_{h}(x, y)$ for any $\lambda>0, \mu>0$. It is clear that $d_{h}$ is not a metric on $\mathcal{C}$ since it does not separate $x$ and $\lambda x$ for any $\lambda>0$. In fact, $d_{h}$ is a metric on the projective space $\mathcal{C} / \mathbf{R}^{+}$.

Yamada [30] gave an alternate definition of the Hilbert metric by supporting hyperplanes. Recall that a convex set $\Omega$ can be represented as $\cap_{\pi(b) \in \mathcal{P}} H_{\pi(b)}$ where $H_{\pi(b)}$ is the half space bounded by a supporting hyperplane $\pi(b)$ of $\Omega$ at the boundary point 
b, containing the convex set $\Omega$. Let $\mathcal{P}$ be the set of all the supporting hyperplanes of $\Omega$ (see Fig. 1(c)). Yamada showed that the Hilbert metric can be represented as:

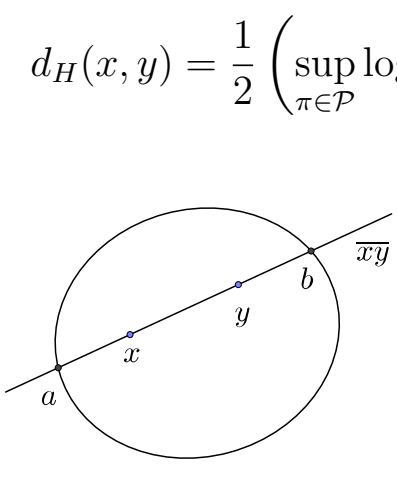

(a)

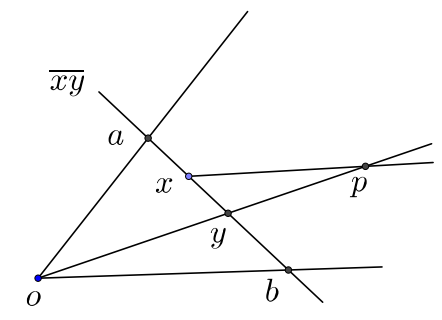

(b)

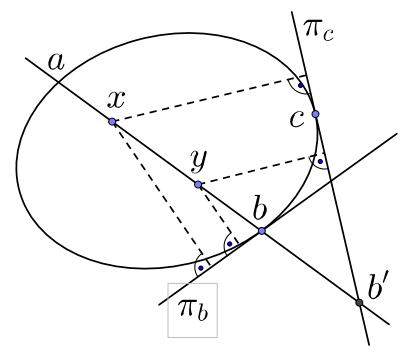

(c)

Figure 1. The Hilbert metric.

We briefly explain Yamada's idea here. Let the line $\overline{x y}$ intersects $\Omega$ at $a, b$ in the order $a, x, y, b$. Let $\pi(b)$ be a supporting hyperplane of $\Omega$ at $b$, and let $\pi(c)$ be a hyperplane of $\Omega$ at an arbitrary point $c \in \Omega$. Denote by $b^{\prime}$ the intersection point between $\overline{x y}$ and $\pi(c)$. It is clear that

$$
\frac{d(x, \pi(c))}{d(y, \pi(c))}=\frac{\left|x-b^{\prime}\right|}{\left|y-b^{\prime}\right|} \leq \frac{|x-b|}{|y-b|}=\frac{d(x, \pi(b))}{d(y, \pi(b))}, \quad \text { for any } c \in \Omega .
$$

Similarly, we have

$$
\frac{d(y, \pi(c))}{d(x, \pi(c))} \leq \frac{d(y, \pi(a))}{d(x, \pi(a))}, \quad \text { for any } c \in \Omega .
$$

Since a cone over a bounded convex set is again a convex set, Yamada's idea also applies to the Birkhoff's version of the Hilbert metric. Therefore

(4) $d_{h}(x, y)=\frac{1}{2}\left(\sup _{\pi \in \mathcal{P}} \log \frac{d(x, \pi)}{d(y, \pi)}+\sup _{\pi \in \mathcal{P}} \log \frac{d(y, \pi)}{d(x, \pi)}\right)$, for any $x, y \in \mathcal{C}$ with $[x] \neq[y]$,

where $[x]=\{\lambda x \in \mathcal{C}: \lambda>0\}$, and $\mathcal{P}$ is the set of supporting hyperplanes of the cone $\mathcal{C}$.

Remark. It is easy to see that these two versions of Hilbert metric coincide on the convex bounded domain $\Omega$, i.e. $d_{H}(x, y)=d_{h}(x, y)$ for any $x, y \in \Omega$. In this paper, we adopt the Birkhoff's version of the Hilbert metric.

2.2. Measured geodesic lamination. Given a reference metric $X_{0}$, a geodesic lamination $L$ is a closed subset of $S_{g, n}$ consisting of mutually disjoint simple geodesics which are called leaves of this geodesic lamination. A transverse invariant measure 
$\mu$ of a geodesic lamination $L$ is a Radon measure defined on every arc $k$ transverse to the support of $L$ such that $\mu$ is invariant with respect to any homotopy of $k$ relative to the leaves of $L$. A measured geodesic lamination is a lamination $L$ endowed with a transverse invariant measure $\mu$. The simplest example of a measured geodesic lamination is a simple closed geodesic, where the transverse invariant measure is the Dirac measure. Each measured geodesic lamination $\mu$ induces a functional on the space $\mathcal{S}$ of isotopy classes of nontrival simple closed curves on $S_{g, n}$, which assigns $\inf _{\tilde{\gamma} \in[\gamma]} \int_{\tilde{\gamma}} d \mu$ to any $[\gamma] \in \mathcal{S}$. The amount $\inf _{\tilde{\gamma} \in[\gamma]} \int_{\tilde{\gamma}} d \mu$ is called the intersection number of $\mu$ with $[\gamma]$ and is denoted by $i(\mu,[\gamma])$. Two measured geodesic laminations $\mu, \mu^{\prime}$ are called equivalent if $i(\mu,[\gamma])=i\left(\mu^{\prime},[\gamma]\right)$ for any $[\gamma] \in \mathcal{S}$. Denote by $\mathcal{M L}$ the space of equivalent classes of measured geodesic laminations on $S_{g, n}$, and equip $\mathcal{M L}$ with the weak topology of the functional space over $\mathcal{S}$. With this topology, the set of weighted simple closed curves, $\mathbf{R}^{+} \times \mathcal{S}$, is dense in $\mathcal{M L}$. The Thurston boundary $\partial T_{g, n}$ of the Teichmüller space $T_{g, n}$ consists of the projective classes of measured geodesic laminations and is homeomorphic to the unit sphere $\mathbf{S}^{6 g-6+2 n}$ (for more details about measured geodesic laminations we refer to [3] and [17]).

2.3. Geometric parametrization of the Teichmüller space. Hamenstädt [5] gave a geometric parametrization of the Teichmüller space $T_{g, m}$. We briefly recall this parametrization. Let $n \geq 1$ and let $X \in T_{g, n}$. Fix one of the punctures of $X$ and denote it by $O$. As we explained in the introduction, a preferred triangulation $\Gamma$ is a set of $6 g-5+2 n$ mutually disjoint simple geodesics $\eta_{1}, \ldots, \eta_{6 g-5+2 n}$ on $S$ with the two ends of each simple geodesic going into the puncture $O$ and which decompose $X$ into $4 g-3+n$ ideal triangles and $n-1$ once-punctured discs. The space of measured lamination $\mathcal{M L}(S)$ on $X$ can be parameterized by the $6 g-5+2 n$-tuple $\left(i\left(\eta_{1}, \mu\right), \ldots, i\left(\eta_{6 g-5+2 n}, \mu\right)\right) \in \mathbf{R}^{6 g-5+2 n}$, where $\mu$ is a measured geodesic lamination with compact support and $i\left(\eta_{i}, \mu\right)$ represents the intersection number of $\mu$ with $\eta_{i}$. Let $\mathcal{A}$ be the set of all $6 g-5+2 n$-tuples $\left(a_{1}, \ldots, a_{6 g-5+2 n}\right)$ of nonnegative real numbers with the following properties:

(1) $a_{i} \leq a_{j}+a_{k}$ if the geodesics $\eta_{i}, \eta_{j}, \eta_{k}$ are the sides of an ideal triangle on $S$.

(2) There is at least one ideal triangle on $S$ with sides $\eta_{i}, \eta_{j}, \eta_{k}$ such that $a_{i}=$ $a_{j}+a_{k}$.

In particular, $\mathcal{A}$ is a cone with vertex at the origin over the boundary of a convex finite-sided polyhedron $P$ in the sphere $\mathbf{S}^{6 g-6+2 n}$. And $\mathcal{A}$ is homeomorphic to $\mathbf{R}^{6 g-6+2 n}$.

Theorem 2.1. [5] The map $\mu \in \mathcal{M L}(S) \rightarrow\left(i\left(\eta_{1}, \mu\right), \ldots, i\left(\eta_{6 g-5+2 n}, \mu\right)\right) \in$ $\mathbf{R}^{6 g-5+2 n}$ is a homeomorphism of $\mathcal{M L}(S)$ onto $\mathcal{A}$.

Since for any marked hyperbolic metric $X \in T_{g, m}$, the length of $\eta_{i}$ is infinite for any $i=1,2, \ldots, 6 g-5+2 n$, we need a little modification to parameterize the Teichmüller space $T_{g, m}$. Recall that every puncture of $S$ admits a standard cusp neighbourhood which is isometric to a cylinder $[-\log 2, \infty) \times S^{1}$ equipped with the metric $d \rho^{2}+e^{-2 \rho} d t^{2}([2])$. The $\rho$-coordinate in this representation is called the height. Let $\Delta_{\infty}$ be an ideal triangle on the upper half plane (see Fig. 2) with sides $\eta_{1}, \eta_{2}, \eta_{3}$. Each corner of $\Delta_{\infty}$ can be foliated by horocycles. Extend these foliations until they fill all but in the center bounded by three horocycles $M_{1} M_{2}, M_{2} M_{3}, M_{3} M_{2}$. We call $M_{i}$ the midpoint of $\eta_{i}$ with respect to the ideal triangle $\Delta_{\infty}$ for $i=1,2,3$. Choose the height $\rho_{0}$ small enough such that $e^{-\rho_{0}}$ is much smaller than the length of the horocycle $M_{1} M_{2}$ (note that the three horocycles $M_{1} M_{2}, M_{2} M_{3}, M_{3} M_{2}$ have the same length). 
Every geodesic going into the cusp meets the horocycles $\rho=$ constant orthogonally. Hence each choice of a height $\rho_{0}$ cuts from $\eta_{i}$ a unique compact arc of finite length since both ends of the geodesic $\eta_{i}$ go into the cusp for any $i=1,2, \ldots, 6 g-5+2 n$. Denote by $l_{\eta_{i}}(X)$ the length of this subarc of $\eta_{i}$ for a marked hyperbolic metric $X$ for any $i=1,2, \ldots, 6 g-5+2 n$.

Theorem 2.2. [5] Let $\Pi: \mathbf{R}^{6 g-5+2 n} \backslash\{0\} \rightarrow \mathbf{R} P^{6 g-6+2 n}$ be the canonical projection. The map

$$
\Lambda: S \in T_{g, n} \rightarrow\left(l_{\eta_{1}}(S), \ldots, l_{\eta_{6 g-5+2 n}}(S)\right) \in \mathbf{R}^{6 g-5+2 n}
$$

is a diffeomorphism of $T_{g, n}$ onto a hypersurface in $\mathbf{R}^{6 g-5+2 n}$. And the map $\Pi \circ \Lambda$ is a diffeomorphism of $T_{g, n}$ onto the interior of a finite-sided closed convex polyhedron $P$ in $\mathbf{R} P^{6 g-6+2 n}$ which extends to a homeomorphism of $T_{g, n} \cup \partial T_{g, n}$ onto $P$.

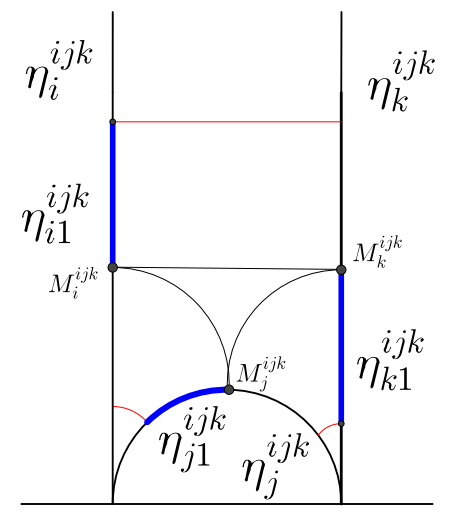

Figure 2. The ideal triangle $\Delta_{\infty}$.

2.4. The Hilbert metric on the Teichmüller space. Now we give the Hilbert metric on the Teichmüller space. Let $\pi_{i j k}$ be the hyperplane in $\mathbf{R}^{6 g-5+2 n}=$ $\left\{\left(a_{1}, a_{2}, \ldots, a_{6 g-5+2 n}\right): a_{i} \in \mathbf{R}\right\}$ defined by

$$
\pi_{i j k}: a_{i}-a_{j}+a_{k}=0,
$$

where the indices $i, j, k$ satisfy the condition that the corresponding simple geodesics $\eta_{i}, \eta_{j}, \eta_{k}$ bound an ideal triangle on the surface. Let $\mathcal{P}$ be the set of all such hyperplanes. By Theorem 2.1 and Theorem 2.2, we know that the image of $T_{g, n} \cup \partial T_{g, n}$ under the map $\Pi \circ \Lambda$ is a polyhedron $P$. Following Yamada's idea, we define the Hilbert metric on $T_{g, n}$ by (4):

$$
\begin{aligned}
d_{h}^{\Gamma}\left(X_{1}, X_{2}\right) & :=d_{h}\left(\Lambda\left(X_{1}\right), \Lambda\left(X_{2}\right)\right) \\
& =\frac{1}{2}\left(\sup _{\pi \in \mathcal{P}} \frac{d\left(\Lambda\left(X_{1}\right), \pi_{i j k}\right)}{d\left(\Lambda\left(X_{2}\right), \pi_{i j k}\right)}+\sup _{\pi \in \mathcal{P}} \frac{d\left(\Lambda\left(X_{2}\right), \pi_{i j k}\right)}{d\left(\Lambda\left(X_{1}\right), \pi_{i j k}\right)}\right),
\end{aligned}
$$

for all $X_{1}, X_{2} \in T_{g, n}$, where $d($,$) represents the Euclidean distance. From (5), we$ have

Hence

$$
d\left(\Lambda(X), \pi_{i j k}\right)=\frac{l_{\eta_{i}}(X)-l_{\eta_{j}}(X)+l_{\eta_{i}}(X)}{3^{1 / 2}} .
$$

$$
\begin{aligned}
d_{h}^{\Gamma}\left(X_{1}, X_{2}\right)= & \frac{1}{2}\left(\sup _{\pi_{i j k} \in \mathcal{P}} \log \frac{l_{\eta_{i}}\left(X_{1}\right)-l_{\eta_{j}}\left(X_{1}\right)+l_{\eta_{k}}\left(X_{1}\right)}{l_{\eta_{i}}\left(X_{2}\right)-l_{\eta_{j}}\left(X_{2}\right)+l_{\eta_{k}}\left(X_{2}\right)}\right. \\
& \left.+\sup _{\pi_{i j k} \in \mathcal{P}} \log \frac{l_{\eta_{i}}\left(X_{2}\right)-l_{\eta_{j}}\left(X_{2}\right)+l_{\eta_{k}}\left(X_{2}\right)}{l_{\eta_{i}}\left(X_{1}\right)-l_{\eta_{j}}\left(X_{1}\right)+l_{\eta_{k}}\left(X_{1}\right)}\right) .
\end{aligned}
$$


Now we give some explanations for (7). Since the surface $S_{g, n}$ is orientable, we fix an orientation. Denote by $\Delta_{i j k}$ the ideal triangle with three sides $\left\{\eta_{i}, \eta_{j}, \eta_{k}\right\}$ such that $\eta_{i}, \eta_{j}, \eta_{k}$ appears in the counterclockwise order. Obviously $\Delta_{i j k}=\Delta_{j k i}=\Delta_{k i j}$. Let $\mathbf{T}_{\Gamma}$ be the collection of all such triangles corresponding to $\Gamma$. The two ends of each of $\eta_{i}, \eta_{j}, \eta_{k}$ go into the cusp $O$ and the intersection of the horocycle $\rho=\rho_{0}$ with the ideal triangle $\Delta_{i j k}$ consists of three components, denoted as $h_{i}^{i j k}, h_{j}^{i j k}, h_{l}^{i j k}$ (the red lines in Fig. 2). It is clear that the sum of the lengths $l_{h_{i}^{i j k}}(X)+l_{h_{j}^{i j k}}(X)+l_{h_{k}^{i j k}}(X)$ over all these $4 g-3+n$ ideal triangles is less than the length of the horocycle $\rho=\rho_{0}$. Denote by $M_{i}^{i j k}$ the midpoint of $\eta_{i}$ with respect to the ideal triangle $\Delta_{i j k} . M_{i}$ together with $h_{j}^{i j k}, h_{k}^{i j k}$ cuts $\eta_{i}$ into four connected components, two of which are compact, denoted by $\eta_{i 1}^{i j k}$ and $\eta_{i 2}^{i j k}$ with respect to the counterclockwise order (see Fig. 2). It follows that

$$
l_{\eta_{i 2}^{i j k}}(X)=l_{\eta_{j 1}^{i j k}}(X) ; \quad l_{\eta_{j 2}^{i j k}}(X)=l_{\eta_{k 1}^{i j k}}(X) ; \quad l_{\eta_{k 2}^{i j k}}(X)=l_{\eta_{i 1}^{i j k}}(X) .
$$

Hence

$$
\begin{aligned}
& l_{\eta_{i}}(X)-l_{\eta_{j}}(X)+l_{\eta_{k}}(X)=2 l_{\eta_{i 1}^{i j k}}(X)=2 l_{\eta_{k 2}^{i j k}}(X), \\
& l_{\eta_{j}}(X)-l_{\eta_{k}}(X)+l_{\eta_{i}}(X)=2 l_{\eta_{j 1}^{i j k}}(X)=2 l_{\eta_{i 2}^{i j k}}(X), \\
& l_{\eta_{k}}(X)-l_{\eta_{i}}(X)+l_{\eta_{j}}(X)=2 l_{\eta_{k 1}^{i j k}}(X)=2 l_{\eta_{j 2}^{i j k}}(X) .
\end{aligned}
$$

Now (7) can be rewritten as

$$
\begin{aligned}
d_{h}^{\Gamma}\left(X_{1}, X_{2}\right)= & \frac{1}{2} \sup _{\Delta_{i j k} \in \mathbf{T}_{\Gamma}} \max \left\{\log \frac{l_{\eta_{i 1}^{i j k}}\left(X_{1}\right)}{l_{\eta_{i 1}^{i j k}}\left(X_{2}\right)}, \log \frac{l_{\eta_{j 1}^{i j k}}\left(X_{1}\right)}{l_{\eta_{j 1}^{i j k}}\left(X_{2}\right)}, \log \frac{l_{\eta_{k 1}^{i j k}}\left(X_{1}\right)}{l_{\eta_{k 1}^{i j k}}\left(X_{2}\right)}\right\} \\
& +\frac{1}{2} \sup _{\Delta_{i j k} \in \mathbf{T}_{\Gamma}} \max \left\{\log \frac{l_{\eta_{i 1}^{i j k}}\left(X_{2}\right)}{l_{\eta_{i 1}^{i j k}}\left(X_{1}\right)}, \log \frac{l_{\eta_{j 1}^{i j k}}\left(X_{2}\right)}{l_{\eta_{j 1}^{i j k}}\left(X_{1}\right)}, \log \frac{l_{\eta_{k 1}^{i j k}}\left(X_{2}\right)}{l_{\eta_{k 1}^{i j k}}\left(X_{1}\right)}\right\} .
\end{aligned}
$$

And the length of the horocycle $h_{i}^{i j k}$ can be expressed in terms of $l_{\eta_{j 2}^{i j k}}$ and $l_{\eta_{k 1}^{i j k}}$ as $l_{h_{i}^{i j k}}(X)=\exp \left(-l_{\eta_{k 1}^{i j k}}(X)\right)=\exp \left(-l_{\eta_{j 2}^{i j k}}(X)\right)$. Therefore

$$
l_{\eta_{i 1}^{i j k}} \geq \rho_{0}, l_{\eta_{j 1}^{i j k}} \geq \rho_{0}, l_{\eta_{k 1}^{i j k}} \geq \rho_{0}, \text { for any ideal triangle } \Delta_{i j k} \in \mathbf{T}_{\Gamma}
$$

We summarize our discussions above as the following proposition.

Proposition 2.3. With the notations above, the Hilbert metric $d_{h}^{\Gamma}$ on the Teichmüller space $T_{g, n}$ can be expressed as the following two forms.

- For any $X_{1}, X_{2} \in T_{g, n}$,

$$
\begin{aligned}
d_{h}^{\Gamma}\left(X_{1}, X_{2}\right)= & \frac{1}{2}\left(\sup _{\Delta_{i j k} \in \mathbf{T}_{\Gamma}} \log \frac{l_{\eta_{i}}\left(X_{1}\right)-l_{\eta_{j}}\left(X_{1}\right)+l_{\eta_{k}}\left(X_{1}\right)}{l_{\eta_{i}}\left(X_{2}\right)-l_{\eta_{j}}\left(X_{2}\right)+l_{\eta_{k}}\left(X_{2}\right)}\right. \\
& \left.+\sup _{\Delta_{i j k} \in \mathbf{T}_{\Gamma}} \log \frac{l_{\eta_{i}}\left(X_{2}\right)-l_{\eta_{j}}\left(X_{2}\right)+l_{\eta_{k}}\left(X_{2}\right)}{l_{\eta_{i}}\left(X_{1}\right)-l_{\eta_{j}}\left(X_{1}\right)+l_{\eta_{k}}\left(X_{1}\right)}\right),
\end{aligned}
$$

where, $l_{\eta_{i}}(X)-l_{\eta_{j}}(X)+l_{\eta_{k}}(X) \geq 2 \rho_{0}$ for any $X \in T_{g, n}$ and any ideal triangle $\Delta_{i j k} \in \mathbf{T}_{\Gamma}$. 
- For any $X_{1}, X_{2} \in T_{g, n}$,

$$
\begin{aligned}
d_{h}^{\Gamma}\left(X_{1}, X_{2}\right)= & \frac{1}{2} \sup _{\Delta_{i j k} \in \mathbf{T}_{\Gamma}} \max \left\{\log \frac{l_{\eta_{i 1}^{i j k}}\left(X_{1}\right)}{l_{\eta_{i 1}^{i j k}}\left(X_{2}\right)}, \log \frac{l_{\eta_{j 1}^{i j k}}\left(X_{1}\right)}{l_{\eta_{j 1}^{i j k}}\left(X_{2}\right)}, \log \frac{l_{\eta_{k 1}^{i j k}}\left(X_{1}\right)}{l_{\eta_{k 1}^{i j k}}\left(X_{2}\right)}\right\} \\
& +\frac{1}{2} \sup _{\Delta_{i j k} \in \mathbf{T}_{\Gamma}} \max \left\{\log \frac{l_{\eta_{i 1}^{i j k}}\left(X_{2}\right)}{l_{\eta_{i 1}^{i j k}}\left(X_{1}\right)}, \log \frac{l_{\eta_{j 1}^{i j k}}\left(X_{2}\right)}{l_{\eta_{j 1}^{i j k}}\left(X_{1}\right)}, \log \frac{l_{\eta_{k 1}^{i j k}}\left(X_{2}\right)}{l_{\eta_{k 1}^{i j k}}\left(X_{1}\right)}\right\},
\end{aligned}
$$

where $l_{\eta_{i 1}^{i j k}} \geq \rho_{0}, l_{\eta_{j 1}^{i j k}} \geq \rho_{0}, l_{\eta_{k 1}^{i j k}} \geq \rho_{0}$ for any ideal triangle $\Delta_{i j k} \in \mathbf{T}_{\Gamma}$.

Remark. The reason that we do not consider the Funk metric is that the Funk metric is not projectively invariant while the Hilbert metric is. If we choose a fixed homogenous coordinate for $\mathbf{R} P^{6 g-6+2 n}$, we can define a Funk metric on $T_{g, n}$.

\section{Earthquake}

3.1. Earthquake. For any $X \in T_{g, n}, \beta \in \mathcal{S}$, denote by $l_{\beta}(X)$ the length of the geodesic representative of $\beta$ on $X$. In [9], Kerckhoff proved that for any simple closed curve $\beta, l_{\beta}\left(\mathcal{E}_{\alpha}^{t} X\right)$ is a convex function of $t$ along the earthquake line $\left\{\mathcal{E}_{\alpha}^{t} X\right\}_{t \in \mathbf{R}}$. Based on this result, Bonahon proved [1] that each (anti-)earthquake ray converges to a unique point in the Thurston boundary $\partial T_{g, n}$.

Lemma 3.1. [9] For any $X \in T_{g, n}, \alpha \in \mathcal{M L}$ and $\beta \in \mathcal{S}$, with $i(\alpha, \beta)>0$, then

$$
\frac{d}{d t} l_{\beta}\left(\mathcal{E}_{\alpha}^{t} X\right)=\int_{\beta} \cos \theta_{t} d \alpha
$$

where $\theta_{t}$ represents the angle at each intersection point between (the geodesic representatives of $\beta$ and $\alpha$ on $\mathcal{E}_{\alpha}^{t} X$ measured counter-clockwise from $\beta$ to $\alpha$. Moreover, as $t$ tends to $+\infty$ (resp. $-\infty$ ), the function $t \mapsto \cos \theta_{t}$ is strictly increasing (resp. decreasing).

Lemma 3.2. [1] For every $X \in T_{g, n}$ and every $\alpha \in \mathcal{M L}$,

$$
\lim _{t \rightarrow \pm \infty} \frac{1}{|t|} i\left(\mathcal{E}_{\alpha}^{t} X, \beta\right)=i(\alpha, \beta), \quad \text { for any } \beta \in \mathcal{S} \text {. }
$$

By applying similar arguments as in [9] and [1], we get similar results for $l_{\eta_{i}}\left(\mathcal{E}_{\alpha}^{t} X\right)$ (note that $l_{\eta_{i}}\left(\mathcal{E}_{\alpha}^{t} X\right)$ is the length of a particular compact subarc of $\eta_{i}$ ).

Lemma 3.3. For any $X \in T_{g, n}, \alpha \in \mathcal{M L}$ and $\eta_{i} \in \Gamma$,

$$
\frac{d}{d t} l_{\eta_{i}}\left(\mathcal{E}_{\alpha}^{t} X\right)=\int_{\eta_{i}} \cos \theta_{t} d \alpha,
$$

where $\theta_{t}$ represents the angle at each intersection point between (the geodesic representatives of ) $\eta_{i}$ and $\alpha$ on $\mathcal{E}_{\alpha}^{t} X$ measured counter-clockwise from $\eta_{i}$ to $\alpha$. Moreover, as $t$ tends to $+\infty$ (resp. $-\infty$ ), the function $t \mapsto \cos \theta_{t}$ is strictly increasing (resp. decreasing).

Proof. The proof is exactly the same as that of Lemma 3.1 in [9].

Lemma 3.4. For every $X \in T_{g, n}$ and every $\alpha \in \mathcal{M L}$,

$$
\lim _{t \rightarrow \pm \infty} \frac{1}{|t|} l_{\eta_{i}}\left(\mathcal{E}_{\alpha}^{t} X\right)=i\left(\alpha, \eta_{i}\right), \text { for any } \eta_{i} \in \Gamma \text {. }
$$


Proof. The proof is exactly the same as that of Lemma 3.2 in [1]. Also, it follows from Lemma 3.5.

In fact, we can prove a stronger result.

Lemma 3.5. For every $X \in T_{g, n}$ and every $\alpha \in \mathcal{M L}$,

$$
\left|l_{\eta_{i}}\left(\mathcal{E}_{\alpha}^{t} X\right)-\right| t\left|i\left(\alpha, \eta_{i}\right)\right| \leq l_{\eta_{i}}(X)+e^{-\rho_{0}}, \text { for any } \eta_{i} \in \Gamma \text { and } t \in \mathbf{R},
$$

where $e^{-\rho_{0}}$ represents the length of the horocycle centered at the puncture $O$ with height $\rho_{0}$. Moreover, there is a constant $c_{i}^{+}, c_{i}^{-} \in\left[-l_{\eta_{i}}(X)-e^{-\rho_{0}},+l_{\eta_{i}}(X)+e^{-\rho_{0}}\right]$ such that

$$
\lim _{t \rightarrow+\infty}\left[l_{\eta_{i}}\left(\mathcal{E}_{\alpha}^{t} X\right)-t i\left(\alpha, \eta_{i}\right)\right]=c_{i}^{+}, \lim _{t \rightarrow-\infty}\left[l_{\eta_{i}}\left(\mathcal{E}_{\alpha}^{t} X\right)-|t| i\left(\alpha, \eta_{i}\right)\right]=c_{i}^{-} .
$$

Proof. By symmetry, it suffices to prove the lemma for $t \geq 0$. We start with the case that $\alpha$ is a simple closed geodesic. Let $f_{i}(t) \triangleq l_{\eta_{i}}\left(\mathcal{E}_{\alpha}^{t} X\right)-t i\left(\eta_{i}, \alpha\right)$. We will show that,

$$
\left|f_{i}(t)\right| \leq l_{\eta_{i}}(X)+e^{-\rho_{0}}, \text { for any } \eta_{i} \in \Gamma \text { and } t \geq 0 .
$$

From Lemma 3.3,

$$
\frac{d f_{i}(t)}{d t}<0, \text { for any } t \geq 0 \text {. }
$$

As a consequence, we get our first inequality immediately,

$$
f_{i}(t) \leq f_{i}(0)=l_{\eta_{i}}(X)
$$

By the monotonicity of $f_{i}(t)$, for any positive integer $m$, we have

$$
f_{i}(t) \geq f_{i}\left(m l_{\alpha}(X)\right) \text {, for any } t \in\left[0, m l_{\alpha}(X)\right] .
$$

Therefore, to prove $f_{i}(t) \geq-l_{\eta_{i}}(X)-e^{-\rho_{0}}$ for all $t \geq 0$, it suffices to prove $f_{i}\left(m l_{\alpha}(X)\right) \geq$ $-l_{\eta_{i}}(X)-e^{-\rho_{0}}$ for all $m \in \mathbf{N}^{+}$.

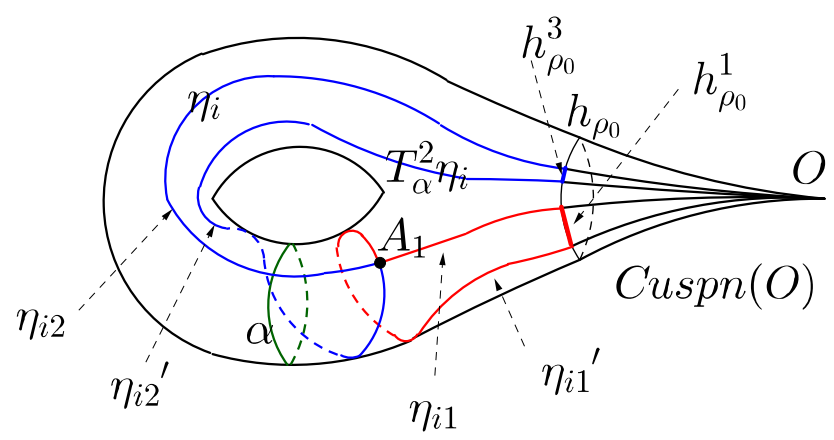

Figure 3. Dehn twist. $T_{\alpha}^{2} \beta$ is obtained from $\beta$ by two times Dehn twist along $\alpha$.

Recall that $\mathcal{E}_{\alpha}^{m l_{\alpha}(X)} X$ is obtained from $X$ by $m$ times Dehn twist along $\alpha$. Denote by $T_{\alpha}^{m}$ the $m$ times Dehn twist along $\alpha$. Hence $l_{\eta_{i}}\left(\mathcal{E}_{\alpha}^{m l_{\alpha}(X)} X\right)=l_{T_{\alpha}^{-m} \eta_{i}}(X)$. Denote by $h_{\rho_{0}}$ the horocycle centered at the puncture $O$ with height $\rho_{0}$, and denote by $\eta_{i}^{*},\left(T_{\alpha}^{-m} \eta_{i}\right)^{*}$ the geodesic representatives of $\eta_{i}, T_{\alpha}^{-m} \eta_{i}$ on $X$, respectively. It is clear that $\eta_{i}^{*}$ and $\left(T_{\alpha}^{-m} \eta_{i}\right)^{*}$ have $m i\left(\eta_{i}, \alpha\right)$ intersection points, one of which is the puncture $O$. The remaining $m i\left(\eta_{i}, \alpha\right)-1$ intersection points cut the restrictions of $\eta_{i}^{*}$ and $\left(T_{\alpha}^{-m} \eta_{i}\right)^{*}$ on $X \backslash \operatorname{Cuspn}(O)$ into $2 m i\left(\eta_{i}, \alpha\right)$ segments $\eta_{i 1}, \eta_{i 1}^{\prime}, \ldots, \eta_{i l}, \eta_{i l}^{\prime}$, where $l=\operatorname{mi}\left(\eta_{i}, \alpha\right)$ and $\operatorname{Cuspn}(O)$ represents the cusp neighbourhood of $O$ with boundary horocycle $h_{\rho_{0}}$. On the other hand, $\eta_{i}^{*}$ and $\left(T_{\alpha}^{-m} \eta_{i}\right)^{*}$ cut the horocycle $h_{\rho_{0}}$ into four segments $h_{\rho_{0}}^{1}, h_{\rho_{0}}^{2}, h_{\rho_{0}}^{3}, h_{\rho_{0}}^{4}$. Two of these four segments, say $h_{\rho_{0}}^{1}, h_{\rho_{0}}^{3}$, together 
with above mentioned $2 m i\left(\eta_{i}, \alpha\right)$ segments $\eta_{i 1}, \eta_{i 1}^{\prime}, \ldots, \eta_{i l}, \eta_{i l}^{\prime}$ consist $m i\left(\eta_{i}, \alpha\right)$ simple closed curves, and all of them are homotopic to $\alpha$ (see Fig. ??). More precisely, let $\gamma_{1}=\eta_{i 1} * \eta_{i 1}^{\prime} * h_{\rho_{0}}^{1}, \gamma_{2}=\eta_{i 2} * \eta_{i 2}^{\prime}, \cdots, \gamma_{l-1}=\eta_{i, l-1} * \eta_{i, l-1}^{\prime}, \gamma_{l}=\eta_{i l} * \eta_{i l}^{\prime} * h_{\rho_{0}}^{3} \cdot \gamma_{1}, \cdots, \gamma_{l}$ are simple closed curves, and all of them are isotopic to $\alpha$. It follows that

$$
m i\left(\eta_{i}, \alpha\right) l_{\alpha}(X) \leq l_{T_{\alpha}^{-m} \eta_{i}}(X)+l_{\eta_{i}}(X)+e^{-\rho_{0}} \text {, for any } m \in \mathbf{N}^{+},
$$

which means that

$$
f_{i}\left(m l_{\alpha}(X)\right) \geq-l_{\eta_{i}}(X)-e^{-\rho_{0}}, \text { for any } m \in \mathbf{N}^{+} .
$$

This completes the proof of (12).

Now we extend these estimates to an arbitrary measured geodesic lamination $\mu$. Recall that $\mathbf{R}^{+} \times \mathcal{S}$ is dense in $\mathcal{M L}$. There exists $\left(s_{m}, \alpha_{m}\right) \in \mathbf{R}^{+} \times \mathcal{S}$ such that $s_{m} \alpha_{m} \rightarrow \mu$, as $m \rightarrow \infty$. It follows from Proposition 1.1 that for any given $t \in \mathbf{R}$, $\mathcal{E}_{s_{m} \alpha_{m}}^{t} X \rightarrow \mathcal{E}_{\mu}^{t} X$ as $m \rightarrow \infty$. Hence for any given $t \in \mathbf{R}, l_{\eta_{i}}\left(\mathcal{E}_{s_{m} \alpha_{m}}^{t} X\right) \rightarrow l_{\eta_{i}}\left(\mathcal{E}_{\mu}^{t} X\right)$ as $m \rightarrow \infty$. From (12), we have

$$
\left|t s_{m} i\left(\alpha_{m}, \eta_{i}\right)-l_{\eta_{i}}\left(\mathcal{E}_{s_{m} \alpha_{m}}^{t} X\right)\right| \leq l_{\eta_{i}}(X)+e^{-\rho_{0}} \text { for any } m \in \mathbf{N}^{+}, \eta_{i} \in \Gamma \text { and } t \geq 0 \text {. }
$$

Let $m$ tend to infinity, we have

$$
\left|l_{\eta_{i}}\left(\mathcal{E}_{\mu}^{t} X\right)-t i\left(\mu, \eta_{i}\right)\right| \leq l_{\eta_{i}}(X)+e^{-\rho_{0}}, \text { for any } \eta_{i} \in \Gamma \text { and } t \geq 0 .
$$

The existence of the $\operatorname{limit} \lim _{t \rightarrow+\infty}\left[l_{\eta_{i}}\left(\mathcal{E}_{\mu}^{t} X\right)-t i\left(\mu, \eta_{i}\right)\right]$ follows from the boundedness and the monotonicity of $l_{\eta_{i}}\left(\mathcal{E}_{\mu}^{t} X\right)-t i\left(\mu, \eta_{i}\right)$.

Based on the estimates in Lemma 3.5, we can describe the coordinates of an earthquake line $\left\{\mathcal{E}_{\mu}^{t} X\right\}_{t \in \mathbf{R}}$. Denote by $P_{1}$ the hyperplane

$$
\left\{\left(x_{1}, x_{2}, \ldots, x_{6 g-5+2 n}\right): x_{1}+x_{2}+\ldots+x_{6 g-5+2 n}=1\right\},
$$

and denote by $\pi$ the projective map

$$
\begin{aligned}
& \pi: \quad \mathbf{R}_{+}^{6 g-5+2 n} \quad \longrightarrow \quad P_{1} \\
& \left(x_{1}, x_{2}, \ldots, x_{6 g-5+2 n}\right) \longmapsto \frac{\left(x_{1}, x_{2}, \ldots, x_{6 g-5+2 n}\right)}{x_{1}+x_{2}+\ldots+x_{6 g-5+2 n}} .
\end{aligned}
$$

For a measured geodesic lamination $\mu$, let $I(\mu)=\sum_{i=1}^{6 g-5+2 n} i\left(\eta_{i}, \mu\right)$. It is clear that $\pi \circ \Lambda(\mu)=I(\mu)^{-1}\left(i\left(\eta_{1}, \mu\right), \ldots, i\left(\eta_{6 g-5+2 n}, \mu\right)\right)$. Moreover, it follows from Lemma 3.5 that for large enough $|t|$,

$$
\pi \circ \Lambda\left(\mathcal{E}_{\mu}^{t} X\right)=\pi \circ \Lambda(\mu)+\frac{1}{|t|} \frac{c_{i}}{I(\mu)}+o\left(\frac{1}{|t|}\right) .
$$

In other words, the images of the earthquake rays $\left\{\mathcal{E}_{\mu}^{t} X\right\}_{t \geq T}$ and $\left\{\mathcal{E}_{\mu}^{t} X\right\}_{t \leq-T}$ in $P_{1}$ look like straight line segments when $T$ is large enough.

Now we prove the main theorem of this paper.

Main Theorem. After reparametrization, every (anti-)earthquake ray is an almost-geodesic in $\left(T_{g, n}, d_{h}^{\Gamma}\right)$.

Proof. By symmetry, it suffices to prove the theorem for every earthquake ray. Let $\left\{\mathcal{E}_{\mu}^{t}(X)\right\}_{t \geq 0}$ be an earthquake ray directed by $\mu$ and starting at $X$. Set $X_{t}=$ $\mathcal{E}_{\mu}^{t}(X)$. Let $f_{i}(t)=l_{\eta_{i}}\left(\mathcal{E}_{\mu}^{t} X\right)-t i\left(\mu, \eta_{i}\right)$. It follows from Lemma 3.5 that

$$
\left|f_{i}(t)\right|<l_{\eta_{i}}(X)+e^{-\rho_{0}},
$$

and that for any $\epsilon>0$ there is a constant $T_{1}>0$ depending on $\epsilon, X, \Gamma$ and $\mu$ such that

$$
\left|f_{i}(t)-c_{i}\right|<\epsilon \text {, for any } t>T_{1}
$$


Moreover, if $i\left(\mu, \eta_{j}\right)=0$ for some $j, l_{\eta_{j}}\left(\mathcal{E}_{\mu}^{t} X\right) \equiv l_{\eta_{j}}(X)$.

Next we divide the set of ideal triangles corresponding to the preferred triangulation $\Gamma$ into three types:

- Type A consists of ideal triangles $\Delta_{i j k}$ which do not intersect $\mu$, i.e. $i\left(\eta_{i}, \mu\right)=$ $i\left(\eta_{j}, \mu\right)=i\left(\eta_{k}, \mu\right)=0$

- Type B consists of ideal triangles $\Delta_{i j k}$ whose two sides, say $\eta_{i}, \eta_{j}$, intersect $\alpha$ and whose remaining side does not intersect $\mu$, i.e. $i\left(\eta_{i}, \mu\right)=i\left(\eta_{j}, \mu\right)>0$ and $i\left(\eta_{k}, \mu\right)=0$;

- Type $\mathrm{C}$ consists of ideal triangles $\Delta_{i j k}$ with each side intersecting $\mu$, i.e. $i\left(\eta_{i}, \mu\right)>0, i\left(\eta_{j}, \mu\right)>0, i\left(\eta_{k}, \mu\right)>0$.

For each triangle $\Delta_{i j k}$, set

$$
d_{i j k}=\frac{c_{i}-c_{j}+c_{k}}{l_{\eta_{i}}\left(X_{0}\right)-l_{\eta_{j}}\left(X_{0}\right)+l_{\eta_{k}}\left(X_{0}\right)} \quad \text { and } \quad \bar{d}_{i j k}=\frac{i\left(\eta_{i}, \mu\right)-i\left(\eta_{j}, \mu\right)+i\left(\eta_{k}, \mu\right)}{l_{\eta_{i}}\left(X_{0}\right)-l_{\eta_{j}}\left(X_{0}\right)+l_{\eta_{k}}\left(X_{0}\right)} .
$$

Now we discuss case by case.

Type A. In this case, $i\left(\eta_{i}, \mu\right)=i\left(\eta_{j}, \mu\right)=i\left(\eta_{k}, \mu\right)=0$.

$$
\frac{l_{\eta_{i}}\left(X_{s}\right)-l_{\eta_{j}}\left(X_{s}\right)+l_{\eta_{k}}\left(X_{s}\right)}{l_{\eta_{i}}\left(X_{t}\right)-l_{\eta_{j}}\left(X_{t}\right)+l_{\eta_{k}}\left(X_{t}\right)} \equiv \frac{l_{\eta_{i}}\left(X_{0}\right)-l_{\eta_{j}}\left(X_{0}\right)+l_{\eta_{k}}\left(X_{0}\right)}{l_{\eta_{i}}\left(X_{0}\right)-l_{\eta_{j}}\left(X_{0}\right)+l_{\eta_{k}}\left(X_{0}\right)} \equiv 1 \text {, for any } s, t \geq 0 \text {. }
$$

Type B. In this case, $i\left(\eta_{i}, \mu\right)=i\left(\eta_{j}, \mu\right)>0$ and $i\left(\eta_{k}, \mu\right)=0$. Recall that for any ideal triangle $\Delta_{i^{\prime} j^{\prime} k^{\prime}}$ (see Proposition 2.3),

$$
\frac{l_{\eta_{i^{\prime}}}\left(X_{s}\right)-l_{\eta_{j^{\prime}}}\left(X_{s}\right)+l_{\eta_{k^{\prime}}}\left(X_{s}\right)}{2} \geq \rho_{0}>0 .
$$

Hence for any $\epsilon>0$, there is a constant $T_{2}>0$ depending on $\epsilon, \Gamma, X$ and $\mu$ such that for any $s, t>T_{2}$, we have

$$
\begin{aligned}
& \frac{l_{\eta_{i}}\left(X_{s}\right)-l_{\eta_{j}}\left(X_{s}\right)+l_{\eta_{k}}\left(X_{s}\right)}{l_{\eta_{i}}\left(X_{t}\right)-l_{\eta_{j}}\left(X_{t}\right)+l_{\eta_{k}}\left(X_{t}\right)}=\frac{f_{i}(s)-f_{j}(s)+l_{\eta_{k}}(X)}{f_{i}(t)-f_{j}(t)+l_{\eta_{k}}(X)} \in(1-\epsilon, 1+\epsilon), \\
& \frac{l_{\eta_{k}}\left(X_{s}\right)-l_{\eta_{i}}\left(X_{s}\right)+l_{\eta_{j}}\left(X_{s}\right)}{l_{\eta_{k}}\left(X_{t}\right)-l_{\eta_{i}}\left(X_{t}\right)+l_{\eta_{j}}\left(X_{t}\right)} \in(1-\epsilon, 1+\epsilon),
\end{aligned}
$$

and

$$
\begin{aligned}
\frac{l_{\eta_{j}}\left(X_{s}\right)-l_{\eta_{k}}\left(X_{s}\right)+l_{\eta_{i}}\left(X_{s}\right)}{l_{\eta_{j}}\left(X_{t}\right)-l_{\eta_{k}}\left(X_{t}\right)+l_{\eta_{i}}\left(X_{t}\right)} & =\frac{i\left(\eta_{j}, \mu\right) s+i\left(\eta_{i}, \mu\right) s+f_{i}(s)+f_{j}(s)-l_{\eta_{k}}(X)}{i\left(\eta_{j}, \mu\right) t+i\left(\eta_{i}, \mu\right) t+f_{i}(s)+f_{j}(s)-l_{\eta_{k}}(X)} \\
& \in\left((1-\epsilon) \frac{s}{t},(1+\epsilon) \frac{s}{t}\right) .
\end{aligned}
$$

Moreover, there is $T_{2}^{\prime}>0$ depending on $\epsilon, \Gamma, X$ and $\mu$ such that for any $s, t>T_{2}^{\prime}$, we have

$$
\begin{aligned}
\frac{l_{\eta_{i}}\left(X_{s}\right)-l_{\eta_{j}}\left(X_{s}\right)+l_{\eta_{k}}\left(X_{s}\right)}{l_{\eta_{i}}\left(X_{0}\right)-l_{\eta_{j}}\left(X_{0}\right)+l_{\eta_{k}}\left(X_{0}\right)} & =\frac{f_{i}(s)-f_{j}(s)+l_{\eta_{k}}(X)}{l_{\eta_{i}}\left(X_{0}\right)-l_{\eta_{j}}\left(X_{0}\right)+l_{\eta_{k}}\left(X_{0}\right)} \\
& \in\left((1-\epsilon) d_{i j k},(1+\epsilon) d_{i j k}\right), \\
\frac{l_{\eta_{k}}\left(X_{s}\right)-l_{\eta_{i}}\left(X_{s}\right)+l_{\eta_{j}}\left(X_{s}\right)}{l_{\eta_{k}}\left(X_{0}\right)-l_{\eta_{i}}\left(X_{0}\right)+l_{\eta_{j}}\left(X_{0}\right)} & \in\left((1-\epsilon) d_{k i j},(1+\epsilon) d_{k i j}\right),
\end{aligned}
$$

and

$$
\frac{l_{\eta_{j}}\left(X_{s}\right)-l_{\eta_{k}}\left(X_{s}\right)+l_{\eta_{i}}\left(X_{s}\right)}{l_{\eta_{j}}\left(X_{0}\right)-l_{\eta_{k}}\left(X_{0}\right)+l_{\eta_{i}}\left(X_{0}\right)} \in\left((1-\epsilon) s \bar{d}_{j k i},(1+\epsilon) s \bar{d}_{j k i}\right)
$$


where $d_{i j k}$ and $\bar{d}_{i j k}$ are defined by (13).

Type C. In this case, $i\left(\eta_{i}, \mu\right)>0, i\left(\eta_{j}, \mu\right)>0, i\left(\eta_{k}, \mu\right)>0$. We distinguish two subcases.

If $i\left(\eta_{i}, \mu\right)-i\left(\eta_{j}, \mu\right)+i\left(\eta_{k}, \mu\right)>0$, it follow from Lemma 3.5 that there is a constant $T_{3}>0$ depending on $\epsilon, \Gamma, X$ and $\mu$ such that for any $s, t>T_{3}$, we have

$$
\begin{aligned}
\frac{l_{\eta_{i}}\left(X_{s}\right)-l_{\eta_{j}}\left(X_{s}\right)+l_{\eta_{k}}\left(X_{s}\right)}{l_{\eta_{i}}\left(X_{t}\right)-l_{\eta_{j}}\left(X_{t}\right)+l_{\eta_{k}}\left(X_{t}\right)} & =\frac{i\left(\eta_{i}, \mu\right) s-i\left(\eta_{j}, \mu\right) s+i\left(\eta_{k}, \mu\right) s+f_{i}(s)-f_{j}(s)+f_{k}(s)}{i\left(\eta_{i}, \mu\right) t-i\left(\eta_{j}, \mu\right) t+i\left(\eta_{k}, \mu\right) t+f_{i}(s)-f_{j}(s)+f_{k}(s)} \\
& \in\left((1-\epsilon) \frac{s}{t},(1+\epsilon) \frac{s}{t}\right),
\end{aligned}
$$

and

$$
\frac{l_{\eta_{i}}\left(X_{s}\right)-l_{\eta_{j}}\left(X_{s}\right)+l_{\eta_{k}}\left(X_{s}\right)}{l_{\eta_{i}}\left(X_{0}\right)-l_{\eta_{j}}\left(X_{0}\right)+l_{\eta_{k}}\left(X_{0}\right)} \in\left((1-\epsilon) s \bar{d}_{i j k},(1+\epsilon) s \bar{d}_{i j k}\right) .
$$

If $i\left(\eta_{i}, \mu\right)-i\left(\eta_{j}, \mu\right)+i\left(\eta_{k}, \mu\right)=0$, there is a constant $T_{3}^{\prime}>0$ depending on $\epsilon, \Gamma$, $X$ and $\mu$ such that for any $s, t>T_{3}^{\prime}$, we have

$$
\begin{aligned}
\frac{l_{\eta_{i}}\left(X_{s}\right)-l_{\eta_{j}}\left(X_{s}\right)+l_{\eta_{k}}\left(X_{s}\right)}{l_{\eta_{i}}\left(X_{t}\right)-l_{\eta_{j}}\left(X_{t}\right)+l_{\eta_{k}}\left(X_{t}\right)} & =\frac{i\left(\eta_{i}, \mu\right) s-i\left(\eta_{i}, \mu\right) s+i\left(\eta_{k}, \mu\right) s+f_{i}(s)-f_{j}(s)+f_{k}(s)}{i\left(\eta_{i}, \mu\right) t-i\left(\eta_{i}, \mu\right) t+i\left(\eta_{k}, \mu\right) t+f_{i}(s)-f_{j}(s)+f_{k}(s)} \\
& =\frac{f_{i}(s)-f_{j}(s)+f_{k}(s)}{f_{i}(t)-f_{j}(t)+f_{k}(t)} \in(1-\epsilon, 1+\epsilon),
\end{aligned}
$$

and

$$
\frac{l_{\eta_{i}}\left(X_{s}\right)-l_{\eta_{j}}\left(X_{s}\right)+l_{\eta_{k}}\left(X_{s}\right)}{l_{\eta_{i}}\left(X_{0}\right)-l_{\eta_{j}}\left(X_{0}\right)+l_{\eta_{k}}\left(X_{0}\right)} \in\left((1-\epsilon) d_{i j k},(1+\epsilon) d_{i j k}\right) .
$$

Therefore, for any $t \geq s \geq \max \left\{T_{1}, T_{2}, T_{2}^{\prime}, T_{3}, T_{3}^{\prime}\right\}$,

$$
\begin{aligned}
d_{h}^{\Gamma}\left(X_{s}, X_{t}\right)= & \frac{1}{2}\left(\sup _{\Delta_{i j k} \in \mathbf{T}_{\Gamma}} \log \frac{l_{\eta_{i}}\left(X_{t}\right)-l_{\eta_{j}}\left(X_{t}\right)+l_{\eta_{k}}\left(X_{t}\right)}{l_{\eta_{i}}\left(X_{s}\right)-l_{\eta_{j}}\left(X_{s}\right)+l_{\eta_{k}}\left(X_{s}\right)}\right. \\
& \left.+\sup _{\Delta_{i j k} \in \mathbf{T}_{\Gamma}} \log \frac{l_{\eta_{i}}\left(X_{s}\right)-l_{\eta_{j}}\left(X_{s}\right)+l_{\eta_{k}}\left(X_{s}\right)}{l_{\eta_{i}}\left(X_{t}\right)-l_{\eta_{j}}\left(X_{t}\right)+l_{\eta_{k}}\left(X_{t}\right)}\right) \\
\in & \left(\frac{1}{2} \log (1-\epsilon) \frac{t}{s}, \frac{1}{2} \log (1+\epsilon) \frac{t}{s}+\frac{1}{2} \log (1+\epsilon)\right),
\end{aligned}
$$

and

$$
\begin{aligned}
d_{h}^{\Gamma}\left(X_{s}, X_{0}\right)= & \frac{1}{2}\left(\sup _{\Delta_{i j k} \in \mathbf{T}_{\Gamma}} \log \frac{l_{\eta_{i}}\left(X_{s}\right)-l_{\eta_{j}}\left(X_{s}\right)+l_{\eta_{k}}\left(X_{s}\right)}{l_{\eta_{i}}\left(X_{0}\right)-l_{\eta_{j}}\left(X_{0}\right)+l_{\eta_{k}}\left(X_{0}\right)}\right. \\
& \left.+\sup _{\Delta_{i j k} \in \mathbf{T}_{\Gamma}} \log \frac{l_{\eta_{i}}\left(X_{0}\right)-l_{\eta_{j}}\left(X_{0}\right)+l_{\eta_{k}}\left(X_{0}\right)}{l_{\eta_{i}}\left(X_{s}\right)-l_{\eta_{j}}\left(X_{s}\right)+l_{\eta_{k}}\left(X_{s}\right)}\right) \\
\in & \left(\frac{1}{2} \log \frac{(1-\epsilon) s \bar{d}}{(1+\epsilon) d}, \frac{1}{2} \log \frac{(1+\epsilon) s \bar{d}}{(1-\epsilon) d}\right),
\end{aligned}
$$

where $d \triangleq \min \left\{d_{i j k}: i\left(\eta_{i}, \mu\right)-i\left(\eta_{j}, \mu\right)+i\left(\eta_{k}, \mu\right)=0, i\left(\eta_{i}, \mu\right)+i\left(\eta_{j}, \mu\right)+i\left(\eta_{k}, \mu\right)>0\right\}$, and $\bar{d} \triangleq \max \left\{\bar{d}_{i j k}: i\left(\eta_{i}, \mu\right)-i\left(\eta_{j}, \mu\right)+i\left(\eta_{k}, \mu\right)>0\right\}$.

Now, reparametrize the earthquake ray $\left\{\mathcal{E}_{\mu}^{t} X\right\}_{t \geq 0}$ as $\left\{E_{\mu}^{t} X\right\}_{t \geq 0}$ by setting $E_{\mu}^{t} X=$ $\mathcal{E}_{\mu}^{(d / \bar{d}) \exp (2 t)} X$. It follows from (14) and (15) that for any $\epsilon>0$ there is a $T>0$ depending on $\epsilon, \Gamma, X$ and $\mu$ such that

$$
\left|d_{h}^{\Gamma}\left(X, E_{\mu}^{s} X\right)-s\right|<\epsilon, \text { for any } s>T,
$$


and

$$
\left|d_{h}^{\Gamma}\left(E_{\mu}^{t} X, E_{\mu}^{s} X\right)-(t-s)\right|<\epsilon, \text { for any } t \geq s>T .
$$

Therefore $\left\{E_{\mu}^{t} X\right\}_{t \geq 0}$ is an almost geodesic in $\left(T_{g, n}, d_{h}^{\Gamma}\right)$.

3.2. The horofunction boundary of $\left(\boldsymbol{T}_{\boldsymbol{g}, \boldsymbol{n}}, \boldsymbol{d}_{h}^{\Gamma}\right)$. Let $(M, d)$ be a proper geodesic metric space, which is endowed with the topology induced by the metric $d$. We embed $(M, d)$ into $C(M)$, the space of continuous real-valued functions on $X$ endowed with the topology of uniform convergence on bounded sets, by the map below:

$$
\begin{aligned}
h: M & \longrightarrow C(M) \\
z & \longmapsto[M \ni x \mapsto d(x, z)-d(b, z)],
\end{aligned}
$$

where $b \in M$ is a base point. The horofunction boundary of $(M, d)$ is defined to be

$$
\partial{\overline{M_{b}}}^{\text {horo }} \triangleq \overline{h(M)} \backslash h(M)
$$

where $\overline{h(M)}$ represents the closure of $h(M)$ in $C(M)$. The horofunction boundary is independent of the base point, i.e. $\partial \bar{M}_{b}^{\text {horo }}$ is homeomorphic to $\partial{\overline{M_{b^{\prime}}}}^{\text {horo }}$ for $b, b^{\prime} \in M$. A function in $\partial \bar{M}^{\text {horo }}$ is called a horofunction.

Rieffel ([18]) observed that every almost geodesic converges to a unique point in the horofunction boundary of $(X, d)$. Therefore, we have the following corollary.

Corollary 3.6. Every (anti-)earthquake ray converges to a unique point in the horofunction boundary of $\left(T_{g, n}, d_{h}^{\Gamma}\right)$.

Remark. As we mentioned in the beginning of this section, Bonahon proved [1] that each (anti-)earthquake ray converges to a unique point in the Thurston boundary $\partial T_{g, n}$. It follows from Theorem 2.2 that each (anti-)earthquake ray converges to a point in the Euclidean boundary of the polytope $P$. In general, let $D$ be a bounded convex domain in the Euclidean space. Foertsch-Karlsson ([4]) proved that every geodesic under the Hilbert metric converges to a boundary point in $\partial D$ in the Euclidean sense. Walsh ([27]) proved that every sequence converging to a point in the horofunction boundary of the Hilbert geometry converges to a point in the Euclidean boundary $\partial D$. Hence every almost geodesic under the Hilbert metric converges to a boundary point in $\partial D$.

\section{Dependence of $d_{h}^{\Gamma}$ on the preferred triangulation $\Gamma$}

In this section, we investigate the dependence of the Hilbert metric $d_{h}^{\Gamma}$ on the choice of triangulation $\Gamma$. First of all, we define a basic operation for a triangulation, namely, diagonal-flip.

Let $Q$ be an ideal quadrilateral with sides $\eta_{1}, \eta_{2}, \eta_{3}, \eta_{4}$ and ideal vertices (corners) $\mathrm{O}_{1}, \mathrm{O}_{2}, \mathrm{O}_{3}, \mathrm{O}_{4}$. Let $\mathrm{H}_{i}$ be a horocycle around the corner $\mathrm{O}_{i}$ whose length is smaller than $e^{-\rho_{0}}, i=1,2,3,4$, where $\rho_{0}$ is chosen in Section 2.3. Let $\alpha$ be a diagonal geodesic connecting $\mathrm{O}_{2}, \mathrm{O}_{4}$ which triangulates $\mathcal{Q}$ into two triangles $\Delta_{1}$ and $\Delta_{2}$, and $M_{1}, M_{2}, M_{3}, M_{4}$ the "midpoint" of $\eta_{1}, \eta_{2}, \eta_{3}, \eta_{4}$ with respect to these two triangles. Further, let $M_{\alpha}$ and $M_{\alpha}^{\prime}$ be the midpoints of $\alpha$ with respect to $\Delta_{1}$ and $\Delta_{2}$ respectively. Denote by $l_{i 1}$ and $l_{i 2}$ the distances from $M_{i}$ to $H_{i-1}$ and from $M_{i}$ to $H_{i}$ respectively. Let $\beta$ be the other diagonal geodesic, and the notations $M_{1}^{\prime}, M_{2}^{\prime}, M_{3}^{\prime}, M_{4}^{\prime}, M_{\beta}, M_{\beta}^{\prime}, l_{i 1}^{\prime}$ and $l_{i 2}^{\prime}$ are defined similarly. (see Fig. 4.) 
Definition 1. With notations described above, we say that $\beta$ is obtained from $\alpha$ by a diagonal-flip with respect to $Q$. A triangulation $\Gamma^{\prime}$ is said to be obtained from $\Gamma$ by a diagonal-flip if $\Gamma^{\prime} \backslash\left\{\alpha^{\prime}\right\}=\Gamma \backslash\{\alpha\}$ and that $\alpha^{\prime}$ can be obtained from $\alpha$ by a diagonal-flip with respect to some quadrilateral whose four sides are contained in $\Gamma^{\prime} \backslash\left\{\alpha^{\prime}\right\}=\Gamma \backslash\{\alpha\}$.

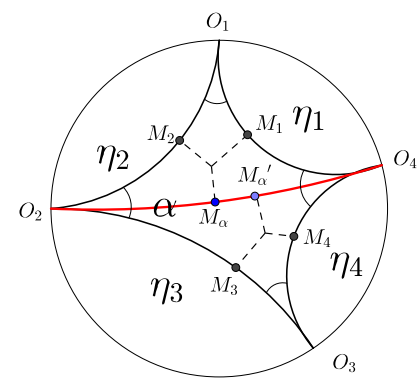

(a)

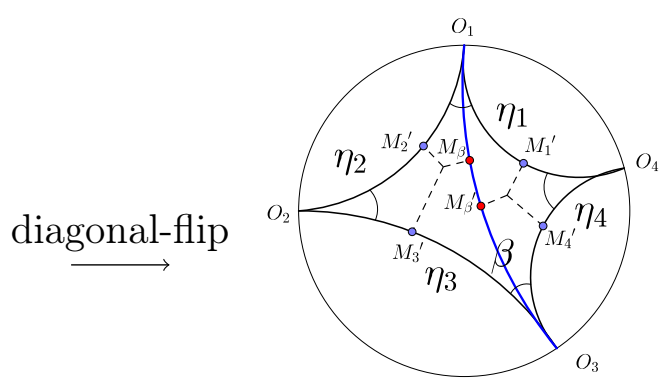

(b)

Figure 4. Flip.

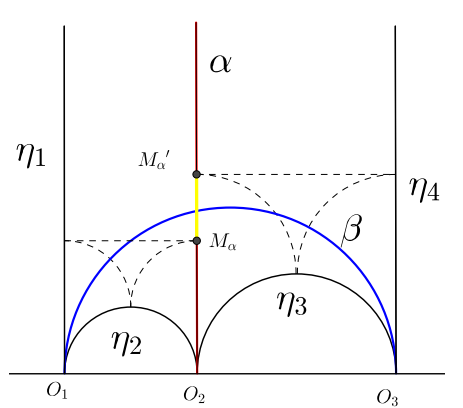

(a)

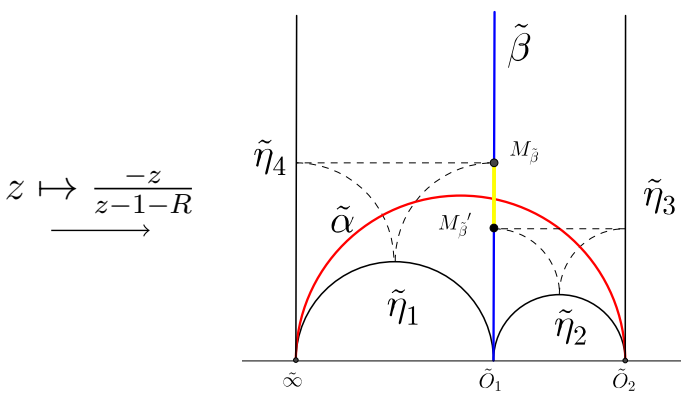

(b)

Figure 5. Shearing.

Definition 2. We define the shearing of $Q$ with respect to $\alpha$, denoted by $\operatorname{shr}(\alpha, Q)$, in the following way. The absolute value of $\operatorname{shr}(\alpha, Q)$ is defined to be the distance between $M_{\alpha}$ and $M_{\alpha}^{\prime}$, and the $\operatorname{sign}$ of $\operatorname{shr}(\alpha, Q)$ is defined to "+" if $M_{\alpha}^{\prime}$ sits on the left side of $M_{\alpha}$ observed from $\Delta_{1}$, otherwise the sign is defined to be "-".

Remark. The "left" notation in the definition of the shearing depends only on the orientation of the ideal quadrilateral.

The lemma below describes some basic properties of a diagonal-flip.

Lemma 4.1. (1) For the shearing of the ideal quadrilateral $Q$ along $\beta, \operatorname{shr}(\beta$; $Q)=-\operatorname{shr}(\alpha ; Q)$.

(2) Suppose $l_{11} \geq l_{42}$, then $\operatorname{shr}(\alpha ; Q)>0$ and

$$
\begin{aligned}
& \left|l_{i 1}^{\prime}-l_{i 1}+\operatorname{shr}(\alpha ; Q)\right| \leq \log 2, \quad\left|l_{i 2}^{\prime}-l_{i 1}-\operatorname{shr}(\alpha ; Q)\right| \leq \log 2, \quad i=1,3 ; \\
& \left|l_{i 1}^{\prime}-l_{i 1}\right| \leq \log 2, \quad\left|l_{i 2}^{\prime}-l_{i 2}\right| \leq \log 2, \quad i=2,4 .
\end{aligned}
$$

Proof. Here we adopt the upper half-plane model for the hyperbolic geometry (see Fig. 5). Since the map $z \mapsto k z$ is an isometry of the hyperbolic metric, we suppose that the Euclidean coordinates of $O_{1}, O_{2}, O_{3}$ are $(0,0),(1,0),(0,1+R)$ respectively. 
(1) The shearing of $Q$ along $\alpha$ is $\log R$. To calculate the shearing of $Q$ along $\beta$, we perform the fractional linear map $f: z \mapsto \frac{-z}{z-1-R}$ on the upper half plane which sends $O_{1}, O_{2}, O_{3}, \infty$ to $(0,0),(1 / R, 0), \infty,-1$ respectively. It is clear in this case that the shearing of $f(Q)$ along $\tilde{\beta}=f(\beta)$ is $\log (1 / R)$, which means the shearing of $Q$ along $\beta$ is also $\log (1 / R)$ since $f$ is an orientation preserving isometry.

(2) By assumption $l_{11} \geq l_{42}$, hence $R \geq 1$. It is clear that $l_{41}+l_{42}=l_{41}^{\prime}+l_{42}^{\prime}$. The coordinates of $M_{4}, M_{4}^{\prime}$ are $(R+1, R)$ and $(1+R, 1+R)$ respectively. In addition, the coordinate of the intersection point between $\eta_{4}$ and $H_{4}$ is $\left(1+R, e^{l_{42}} R\right)$. Therefore $l_{42}^{\prime}=\log \frac{e^{l_{42} R}}{1+R} \in\left[l_{42}-\log 2, l_{42}\right]$, hence $l_{41}^{\prime} \in\left[l_{41}, l_{41}+\log 2\right]$. Similarly we get $l_{22}^{\prime} \in$ $\left[l_{22}-\log 2, l_{22}\right]$ and $l_{21}^{\prime} \in\left[l_{21}, l_{22}+\log 2\right]$.

For the remaining inequalities, note that $l_{12}=l_{21}$ and $l_{11}+l_{12}=l_{11}^{\prime}+l_{42}^{\prime}$, hence $l_{12}^{\prime}=l_{21}^{\prime}-\operatorname{shr}(\beta ; Q) \in\left[l_{12}+\operatorname{shr}(\alpha ; Q), l_{12}+\operatorname{shr}(\alpha ; Q)+\log 2\right]$, and $l_{11}^{\prime} \in\left[l_{11}-\operatorname{shr}(\alpha ; Q)-\right.$ $\left.\log 2, l_{11}-\operatorname{shr}(\alpha ; Q)\right]$. Similarly we get $l_{32}^{\prime} \in\left[l_{32}+\operatorname{shr}(\alpha ; Q), l_{32}+\operatorname{shr}(\alpha ; Q)+\log 2\right]$, and $l_{31}^{\prime} \in\left[l_{31}-\operatorname{shr}(\alpha ; Q)-\log 2, l_{31}-\operatorname{shr}(\alpha ; Q)\right]$.

The estimates from the lemma above describe a close relationship between the changes of the lengths of preferred arcs and the shearing along each simple geodesic $\eta_{i}$ after a diagonal-flip operation. This relationship provides a clue for investigating the effect of the triangulation $\Gamma$ on the Hilbert metric $d_{h}^{\Gamma}$, i.e. investigating the relationship between $d_{h}^{\Gamma}(X, Y)$ and $d_{h}^{\Gamma^{\prime}}(X, Y)$ for two different triangulations $\Gamma, \Gamma^{\prime}$. If we fix a point $X_{0} \in T_{g, n}$, the sphere $B^{\Gamma}\left(X_{0}, R\right)$ centered at $X_{0}$ of radius $R$ with respect to $d_{h}^{\Gamma}$ is an almost-sphere, i.e. a sphere up to an additive constant, with respect to $d_{h}^{\Gamma^{\prime}}$, where $\Gamma^{\prime}$ can be obtained from $\Gamma$ by a diagonal-flip.

Proposition 4.2. Fix $X_{0} \in T_{g, n}$. Let $\Gamma, \Gamma^{\prime}$ be two preferred triangulations of $S_{g, n}$ such that one can be obtained from the other by a diagonal-flip, then there is a constant $C_{\Gamma, \Gamma^{\prime}, X_{0}, \rho_{0}}$ depending on $\Gamma, \Gamma, X_{0}, \rho_{0}$ such that

$$
\left|d_{h}^{\Gamma}\left(X_{0}, X\right)-d_{h}^{\Gamma^{\prime}}\left(X_{0}, X\right)\right| \leq C_{\Gamma, \Gamma^{\prime}, X_{0}, \rho_{0}}, \text { for any } X \in T_{g, n} .
$$

Proof. Set $\Gamma=\left\{\alpha, \eta_{1}, \ldots, \eta_{6 g-6+2 n}\right\}, \Gamma^{\prime}=\left\{\alpha^{\prime}, \eta_{1}, \ldots, \eta_{6 g-6+2 n}\right\}$. First, we prove an inequality which holds for any preferred triangulation $\Gamma$,

$$
\sup _{\eta \in \Gamma \backslash \alpha} l_{\eta}(X) \leq \sup _{\eta \in \Gamma} l_{\eta}(X) \leq 2 \sup _{\eta \in \Gamma \backslash \alpha} l_{\eta}(X), \text { for any } X \in T_{g, n} .
$$

Indeed, there are two simple geodesics $\eta_{i}, \eta_{j} \in \Gamma$ such that $\eta_{i}, \eta_{j}, \alpha$ bound an ideal triangle. Recall that

$$
l_{\eta_{j}}(X)-l_{\alpha}(X)+l_{\eta_{i}}(X) \geq 2 \rho_{0}>0
$$

then

Hence

$$
l_{\alpha}(X) \leq 2 \max \left\{l_{\eta_{i}}(X), l_{\eta_{j}}(X)\right\}
$$

$$
\sup _{\eta \in \Gamma} l_{\eta}(X) \leq 2 \sup _{\eta \in \Gamma \backslash \alpha} l_{\eta}(X) .
$$

The first inequality is obvious. Now the proposition follows immediately from Proposition 4.3 and (16).

Proposition 4.3. Fix $X_{0} \in T_{g, n}$, then there is a constant $C_{\Gamma, X_{0}, \rho_{0}}$ depending on $\Gamma, X_{0}$, and $\rho_{0}$ such that

$$
\left|d_{h}^{\Gamma}\left(X_{0}, X\right)-\frac{1}{2} \sup _{\eta \in \Gamma} \log l_{\eta}(X)\right| \leq C_{\Gamma, X_{0}, \rho_{0}}, \quad \text { for any } X \in T_{g, n} .
$$


Proof. Set $\Gamma=\left\{\eta_{1}, \ldots, \eta_{6 g-5+2 n}\right\}$. By (11),

$$
\begin{aligned}
d_{h}^{\Gamma}\left(X_{1}, X_{2}\right)= & \frac{1}{2}\left(\sup _{\Delta_{i j k} \in \mathbf{T}_{\Gamma}} \log \frac{l_{\eta_{i}}\left(X_{1}\right)-l_{\eta_{j}}\left(X_{1}\right)+l_{\eta_{k}}\left(X_{1}\right)}{l_{\eta_{i}}\left(X_{2}\right)-l_{\eta_{j}}\left(X_{2}\right)+l_{\eta_{k}}\left(X_{2}\right)}\right. \\
& \left.+\sup _{\Delta_{i j k} \in \mathbf{T}_{\Gamma}} \log \frac{l_{\eta_{i}}\left(X_{2}\right)-l_{\eta_{j}}\left(X_{2}\right)+l_{\eta_{k}}\left(X_{2}\right)}{l_{\eta_{i}}\left(X_{1}\right)-l_{\eta_{j}}\left(X_{1}\right)+l_{\eta_{k}}\left(X_{1}\right)}\right) .
\end{aligned}
$$

Note that

$$
2 l_{\eta_{i}}(X)=\left[l_{\eta_{i}}(X)-l_{\eta_{j}}(X)+l_{\eta_{k}}(X)\right]+\left[l_{\eta_{j}}(X)-l_{\eta_{k}}(X)+l_{\eta_{i}}(X)\right]
$$

for any ideal triangle $\Delta_{i j k}$, and

$$
\left[l_{\eta_{i}}(X)-l_{\eta_{j}}(X)+l_{\eta_{k}}(X)\right] \geq 2 \rho_{0}, \text { for any } \Delta_{i j k} .
$$

Therefore

$$
\begin{aligned}
d_{h}^{\Gamma}\left(X_{0}, X\right) & \leq \frac{1}{2}\left[\log \frac{\sup _{\eta \in \Gamma} l_{\eta}(X)}{\rho_{0}}+\log \frac{\sup _{\eta \in \Gamma} l_{\eta}\left(X_{0}\right)}{\rho_{0}}\right] \\
& =\frac{1}{2} \sup _{\eta \in \Gamma} \log l_{\eta}(X)+\frac{1}{2} \sup _{\eta \in \Gamma} \log l_{\eta}\left(X_{0}\right)-\log \rho_{0} .
\end{aligned}
$$

Next we deal with the inverse inequality. Without loss of generality, we assume that $l_{\eta 1}(X)=\sup _{\eta \in \Gamma} l_{\eta}(X)$ and that $\eta_{1}, \eta_{2}, \eta_{3}$ bounds an ideal triangle. By (17), at least one of $l_{\eta_{1}}(X)-l_{\eta_{2}}(X)+l_{\eta_{3}}(X)$ and $l_{\eta_{2}}(X)-l_{\eta_{3}}(X)+l_{\eta_{1}}(X)$ is not less than $\sup _{\eta \in \Gamma} l_{\eta}(X)$. Then

$$
d_{h}^{\Gamma}\left(X_{0}, X\right) \geq \frac{1}{2}\left[\log \frac{\sup _{\eta \in \Gamma} l_{\eta}(X)}{2 \sup _{\eta \in \Gamma} l_{\eta}\left(X_{0}\right)}\right]=\frac{1}{2} \sup _{\eta \in \Gamma} \log l_{\eta}(X)-\frac{1}{2}\left[\log 2+\sup _{\eta \in \Gamma} l_{\eta}\left(X_{0}\right)\right] .
$$

Set $C_{\Gamma, X_{0}, \rho_{0}} \triangleq \max \left\{\frac{1}{2} \sup _{\eta \in \Gamma} \log l_{\eta}\left(X_{0}\right)-\log \rho_{0}, \frac{1}{2} \sup _{\eta \in \Gamma} l_{\eta}\left(X_{0}\right)+\frac{1}{2} \log 2\right\}$, the proposition follows.

\section{Actions of mapping class group}

The mapping class group $M C G\left(S_{g, n}\right)$ consists of the isotopy classes of orientationpreserving self homeomorphisms of $S_{g, n}$. In this section, we study the actions of mapping class group $M C G\left(S_{g, n}\right)$ on the metric space $\left(T_{g, n}, d_{h}^{\Gamma}\right)$. The action is defined as following. For $g \in M C G\left(S_{g, n}\right)$ and $(X, f) \in T_{g, n}, g \circ(X, f)$ is defined as the marked hyperbolic surface $\left(X, f \circ g^{-1}\right)$.

Denote by $P M C G\left(S_{g, n}\right)$ the subgroup of $M C G\left(S_{g, n}\right)$ consisting of elements that fix each puncture individually. It is well known that $P M C G\left(S_{g, n}\right)$ can be generated by finitely many Dehn twists about nonseparating simple closed curves, where a nonseparating simple closed curve $\alpha$ is a closed curve such that $S_{g, n} \backslash \alpha$ is connected (see [2, Chap. 5]).

The lemma below describes the changes of $l_{\eta_{i}}(X)$ under a Dehn twist.

Lemma 5.1. Assume that $\Delta_{123}$ is an ideal triangle on $X \in T_{g, n}$ with three sides $\eta_{1}, \eta_{2}, \eta_{3}$, and that $g$ is a positive Dehn twist of $S_{g, n}$ along an essential simple closed curve $\alpha$. Denote by $\Delta_{123}^{\prime}, \eta_{1}^{\prime}, \eta_{2}^{\prime}, \eta_{3}^{\prime}$, the images of $\Delta_{123}, \eta_{1}, \eta_{2}, \eta_{3}$, respectively, under the action of $g$. Then, there is a constant $C$ depending on the length $l_{\alpha}(X)$, the reference height $\rho_{0}$ and the isotopy classes of $\alpha, \eta_{1}, \eta_{2}, \eta_{3}$ such that

$$
\frac{1}{C} \leq \frac{l_{\eta_{i+1}^{\prime}}(X)+l_{\eta_{i-1}^{\prime}}(X)-l_{\eta_{i}^{\prime}}(X)}{l_{\eta_{i+1}}(X)+l_{\eta_{i-1}}(X)-l_{\eta_{i}}(X)} \leq C, \quad i=1,2,3 .
$$


Proof. Note that

$$
\begin{aligned}
\left|l_{\eta_{i}^{\prime}}(X)-l_{\eta_{i}}(X)\right| & \leq i\left(\eta_{i}, \alpha\right) l_{\alpha}(X), \quad i=1,2,3, \\
i\left(\eta_{i}^{\prime}, \alpha\right) & =i\left(\eta_{i}, \alpha\right), \quad i=1,2,3 .
\end{aligned}
$$

On the other hand, from (10),

$$
\begin{array}{ll}
l_{\eta_{i+1}^{\prime}}(X)+l_{\eta_{i-1}^{\prime}}(X)-l_{\eta_{i}^{\prime}}(X)>2 \rho_{0}, & i=1,2,3 ; \\
l_{\eta_{i+1}}(X)+l_{\eta_{i-1}}(X)-l_{\eta_{i}}(X)>2 \rho_{0}, & j=i-1, i, i+1 .
\end{array}
$$

Hence

$$
\begin{aligned}
\frac{l_{\eta_{i+1}^{\prime}}(X)+l_{\eta_{i-1}^{\prime}}(X)-l_{\eta_{i}^{\prime}}(X)}{l_{\eta_{i+1}}(X)+l_{\eta_{i-1}}(X)-l_{\eta_{i}}(X)} & \leq 1+\frac{\left[i\left(\eta_{i+1}, \alpha\right)+i\left(\eta_{i}, \alpha\right)+i\left(\eta_{i-1}, \alpha\right)\right] l_{\alpha}(X)}{l_{\eta_{i+1}}(X)+l_{\eta_{i-1}}(X)-l_{\eta_{i}}(X)} \\
& \leq 1+\frac{\left[i\left(\eta_{i+1}, \alpha\right)+i\left(\eta_{i}, \alpha\right)+i\left(\eta_{i-1}, \alpha\right)\right] l_{\alpha}(X)}{2 \rho_{0}}
\end{aligned}
$$

Interchange $\eta_{i}$ with $\eta_{i}^{\prime}$, we get the inverse inequality

$$
\frac{l_{\eta_{i+1}}(X)+l_{\eta_{i-1}}(X)-l_{\eta_{i}}(X)}{l_{\eta_{i+1}^{\prime}}(X)+l_{\eta_{i-1}^{\prime}}(X)-l_{\eta_{i}^{\prime}}(X)} \leq 1+\frac{\left[i\left(\eta_{i+1}, \alpha\right)+i\left(\eta_{i}, \alpha\right)+i\left(\eta_{i-1}, \alpha\right)\right] l_{\alpha}(X)}{2 \rho_{0}} .
$$

As an application, we have the following.

Corollary 5.2. Let $\Gamma$ be a preferred triangulation of $S_{g, n}$. Let $g$ be a positive Dehn twist of $S_{g, n}$ along an essential simple closed curve $\alpha$. Set $M_{\alpha, l} \triangleq\left\{X \in T_{g, n}\right.$ : $\left.l_{\alpha}(X) \leq l\right\}$. Then there is a constant $C_{\Gamma, \alpha, \rho, l}$ depending on $\Gamma$, the isotopy class of $\alpha$, the reference height $\rho_{0}$ and $l$ such that

$$
\left|d_{h}^{\Gamma}(X, Y)-d_{h}^{\Gamma}(g X, g Y)\right| \leq C_{\Gamma, \alpha, \rho_{0}, l}, \quad \text { for any } X, Y \in M_{\alpha, l} .
$$

Proof. It follows from Lemma 5.1 that

$$
\begin{aligned}
\frac{1}{C_{\Gamma, \alpha, \rho}} \frac{l_{\eta_{i+1}}(Y)+l_{\eta_{i-1}}(Y)-l_{\eta_{i}}(Y)}{l_{\eta_{i+1}}(X)+l_{\eta_{i-1}}(X)-l_{\eta_{i}}(X)} & \leq \frac{l_{\eta_{i+1}}(g Y)+l_{\eta_{i-1}}(g Y)-l_{\eta_{i}}(g Y)}{l_{\eta_{i+1}}(g X)+l_{\eta_{i-1}}(g X)-l_{\eta_{i}}(g X)} \\
& \leq C_{\Gamma, \alpha, \rho_{0}} \frac{l_{\eta_{i+1}}(Y)+l_{\eta_{i-1}}(Y)-l_{\eta_{i}}(Y)}{l_{\eta_{i+1}}(X)+l_{\eta_{i-1}}(X)-l_{\eta_{i}}(X)},
\end{aligned}
$$

where $C_{\Gamma, \alpha, \rho_{0}}=\left[1+\left(l / \rho_{0}\right) \sum_{i=1}^{6 g-5+2 n} i\left(\eta_{i}, \alpha\right)\right]\left[1+\left(l / \rho_{0}\right) \sum_{i=1}^{6 g-5+2 n} i\left(\eta_{i}, \alpha\right)\right]$.

We do not know whether or not the action of $M C G\left(S_{g, n}\right)$ on $\left(T_{g, n}, d_{h}^{\Gamma}\right)$ is quasiisometric. But for any given $X, Y \in T_{g, n}$, we have the following asymptotic behaviour.

Proposition 5.3. Let $\Gamma$ be a preferred triangulation, and $g \in M C G\left(S_{g, n}\right)$ be a positive Dehn twist about a simple closed curve $\alpha$. For any given $X, Y \in T_{g, n}$, there is a positive number $C_{X, Y}$ depending on $X, Y$ such that

$$
\lim _{n \rightarrow \infty} d_{h}^{\Gamma}\left(g^{n} X, g^{n} Y\right)=C_{X, Y}
$$

Moreover, for any $X \in T_{g, n}$,

$$
\lim _{n \rightarrow \infty} d_{h}^{\Gamma}\left(g^{n} X, g^{n+1} X\right)=0 .
$$

Proof. Note that a Dehn twist is also an earthquake map, i.e. $g=\mathcal{E}_{\alpha}^{l_{\alpha}(X)}$. The remaining discussion is similar to the proof of the Main Theorem.

It follows immediately from Proposition 5.3 that $\left(T_{g, n}, d_{h}^{\Gamma}\right)$ is not $M C G\left(S_{g, n}\right)$ invariant. More precisely, we have the following corollary. 
Corollary 5.4. Let $\Gamma$ be a preferred triangulation, and $g \in M C G\left(S_{g, n}\right)$ be a positive Dehn twist about a simple closed curve $\alpha$. Then the action of $g$ on $T_{g, n}$ is not isometric. In particular, $\left(T_{g, n}, d_{h}^{\Gamma}\right)$ is not $M C G\left(S_{g, n}\right)$ invariant.

\section{References}

[1] Bonahon, F.: Earthquakes on Riemann surfaces and on measured geodesic laminations. Trans. Amer. Math. Soc. 330:1, 1992, 69-95.

[2] Buser, P.: Geometry and spectra of compact Riemann surfaces. - Progr. Math. 106, Birkhäuser Boston, Inc., Boston, MA, 1992.

[3] Fathi, A., F. Laudenbach, and V. Pónnaru: Thurston's work on surfaces. - Math. Notes 48, Princeton Univ. Press, Princeton, NJ, 2012.

[4] Foertsch, T., and A. Karlsson: Hilbert metrics and Minkowski norms. - J. Geom. 83:1-2, $2005,22-31$.

[5] HamenstäDt, U.: Length functions and parameterizations of Teichmüller space for surfaces with cusps. - Ann. Acad. Sci. Fenn. Math. 28:1, 2003, 75-88.

[6] Hamenstädt, U.: Parametrizations of Teichmüller space and its Thurston boundary. - In: Geometric analysis and nonlinear partial differential equations, Springer, Berlin, 2003, 81-88.

[7] DE LA HARPe, P.: On Hilbert's metric for simplices. - In: Geometric group theory, Vol. 1 (Sussex, 1991), London Math. Soc. Lecture Note Ser. 181, Cambridge Univ. Press, Cambridge, 1993, 97-119.

[8] Karlsson, A., V. Metz, and G. A. Noskov: Horoballs in simplices and Minkowski spaces. - Int. J. Math. Math. Sci. 2006, Art. ID 23656, 1-20.

[9] Kerchkoff, S. P.: The Nielsen realization problem. - Ann. of Math. (2) 117, 1983, 235-265.

[10] Kohlberg, E., and J.W. Pratt: The contraction mapping approach to the PerronFrobenius theory: why Hilbert's metric? - Math. Oper. Res. 7:2, 1982, 198-210.

[11] Kohlberg, E., and A. Neyman: Convergence in Hilbert's metric and convergence in direction. - J. Math. Anal. Appl. 93:1, 1983, 104-108.

[12] Lemmens, B., and R. Nussbaum: Birkhoff's version of Hilbert's metric and its applications in analysis. - In: Handbook of Hilbert geometry, IRMA Lect. Math. Theor. Phys. 22, Eur. Math. Soc., Zürich, 2014, 275-303.

[13] Lemmens, B., and C. Walsh: Isometries of polyhedral Hilbert geometries. - J. Topol. Anal. $3: 2,2011,213-241$.

[14] Lins, B.: A Denjoy-Wolff theorem for Hilbert metric nonexpansive maps on polyhedral domains. - Math. Proc. Cambridge Philos. Soc. 143, 2007, 157-164.

[15] Papadopoulos, A.: Problem 13 in problem session "Teichmüller theory". - In: Oberwolfach Reports, Vol. 7, Issue 4, Eur. Math. Soc., Zürich, 2010, 3085-3157.

[16] Papadopoulos, A., and M. Troyanov: From Funk to Hilbert geometry. - In: Handbook of Hilbert geometry, IRMA Lect. Math. Theor. Phys. 22, Eur. Math. Soc., Zürich, 2014, 33-67.

[17] Penner, R. C., and J. L. Harer: Combinatorics of train tracks. - Ann. of Math. Stud. 125, Princeton Univ. Press, Princeton, NJ, 1992.

[18] RiEfFel, M. A.: Group $C^{*}$-algebras as compact quantum metric spaces. - Doc. Math. 7, 2002, 605-651.

[19] Schmutz, S. P.: A cell decomposition of Teichmüller space based on geodesic length functions. - Geom. Funct. Anal. 11:1, 2001, 142-174.

[20] Schmutz, S. P.: A systolic geometric cell decomposition for the space of once-holed Riemann surfaces of genus 2. - Topology 40:5, 2001, 1017-1049.

[21] Seppälä, M., and T. Sorvali: On geometric parametrization of Teichmüller spaces. - Ann. Acad. Sci. Fenn. Ser. A I Math. 10, 1985, 515-526. 
[22] Seppëlä, M., and T. Sorvali: Parametrization of Teichmüller spaces by geodesic length functions. - In: Holomorphic functions and moduli, Vol. II (Berkeley, CA, 1986), Math. Sci. Res. Inst. Publ., 11, Springer, New York, 1988, 267-284.

[23] Thurston, W. P.: Earthquakes in 2-dimensional hyperbolic geometry. - In: Fundamentals of hyperbolic geometry: selected expositions, London Math. Soc. Lecture Note Ser. 328, Cambridge Univ. Press, Cambridge, 2006, 267-289.

[24] Thurston W.P.: Minimal stretch maps between hyperbolic surfaces. - arXiv:math/ $9801039 \mathrm{v} 1$.

[25] Vernicos, C.: On the Hilbert geometry of convex polytopes. - In: Handbook of Hilbert geometry, IRMA Lect. Math. Theor. Phys. 22, Eur. Math. Soc., Zürich, 2014, 111-125.

[26] Vernicos, C.: Lipschitz characterisation of polytopal Hilbert geometries. - Osaka J. Math. $52: 1,2015,215-237$.

[27] Walsh, C.: The horofunction boundary of the Hilbert geometry. - Adv. Geom. 8:4, 2008, $503-529$.

[28] Wu, Y.: Whirly 3-interval exchange transformations. - arXiv:1403.6568.

[29] YAmadA, S.: Weil-Petersson geometry of Teichmüller-Coxeter complex and its finite rank property. - Geom. Dedicata 145, 2010, 43-63.

[30] Yamada, S.: Convex bodies in Euclidean and Weil-Petersson geometries. - Proc. Amer. Math. Soc. 142:2, 2014, 603-616.

Received 30 September 2015 • Accepted 15 January 2016 